\title{
Nucleon electromagnetic and axial-vector form factors.
}

\author{
Harleen Dahiya ${ }^{* \dagger}$ \\ Department of Physics, Dr. B.R. Ambedkar National Institute of Technology, Jalandhar, 144011, \\ India \\ E-mail: dahiyahenitj.ac.in
}

\section{Monika Randhawa}

University Institute of Engineering and Technology, Panjab University, Chandigarh, 160014, India

E-mail: monika@pu.ac.in

The vector and axial-vector nucleon form factors $\left(G_{E, M}^{p, n}\left(Q^{2}\right)\right.$ and $\left.G_{A}^{p, n}\left(Q^{2}\right)\right)$ using the spin observables in the chiral constituent quark model $(\chi \mathrm{CQM})$ have been evaluated in light of the improved precision of the experimental measurements and enormous theoretical progress to understand the dynamical behavior of nucleons emerging from the theory of strong interactions between quarks. The $Q^{2}$ dependence of the vector and axial-vector form factors of the nucleon has been studied using the conventional dipole form of parametrization.

XXV International Workshop on Deep-Inelastic Scattering and Related Subjects

3-7 April 2017

University of Birmingham, $U K$

* Speaker.

${ }^{\dagger}$ Author would like to thank Department of Science and Technology, Government of India for financial support. 
One of the fundamental goals of particle physics is to understand the flavor-spin structure and behavior of strongly interacting matter in terms of its basic constituents, quarks and gluons. Confinement has limited our knowledge on the composition of hadrons and the understanding of their internal structure continues to remain a major unresolved problem in high energy spin physics. The deep inelastic scattering (DIS) experiments with polarized beams and/or targets is an useful probe of calculating the spin carried by the quarks in the nucleon $[1,2,3,4]$. The electromagnetic form factors are among the most basic quantities containing information about the internal structure of the nucleons. Further, during the last few years, the standard electroweak theory has provided a firm basis for the role of weak interaction as a precision probe of the nucleon structure [5, 6, 7].

The hadronic matrix element of the electromagnetic current operators for a spin- $\frac{1}{2}$ nucleon with internal structure can be expressed in terms of the Dirac and Pauli form factors $F_{1}^{N, \gamma}$ and $F_{2}^{N, \gamma}$ $(N=p, n)$ which are further related to the electric and magnetic Sachs form factors $G_{E}^{N, \gamma}\left(Q^{2}\right)$ and $G_{M}^{N, \gamma}\left(Q^{2}\right)$. The quark flavor structure of these form factors can be revealed from the matrix elements of individual quark currents in terms of form factors $F_{1}^{q}$ and $F_{2}^{q}(j=u, d$, or $s)$. The nucleon form factors can be expressed in terms of the individual quark form factors with the electric charge of individual quarks as the coupling constants.

The hadronic matrix element of the neutral weak current operators for a spin- $\frac{1}{2}$ nucleon can be expressed in terms of the vector form factors $F_{1}^{N, Z}$ and $F_{2}^{N, Z}$ as well as the axial form factor $G_{A}^{N, Z}$ which can be expressed in terms of the individual quark form factors as

$$
\begin{aligned}
& G_{E, M}^{p, Z}\left(Q^{2}\right)=\left(1-\frac{8}{3} \sin ^{2} \theta_{W}\right) G_{E, M}^{u}\left(Q^{2}\right)+\left(-1+\frac{4}{3} \sin ^{2} \theta_{W}\right)\left(G_{E, M}^{d}\left(Q^{2}\right)+G_{E, M}^{s}\left(Q^{2}\right)\right), \\
& G_{E, M}^{n, Z}\left(Q^{2}\right)=\left(1-\frac{8}{3} \sin ^{2} \theta_{W}\right) G_{E, M}^{d}\left(Q^{2}\right)+\left(-1+\frac{4}{3} \sin ^{2} \theta_{W}\right)\left(G_{E, M}^{u}\left(Q^{2}\right)+G_{E, M}^{s}\left(Q^{2}\right)\right) .
\end{aligned}
$$

The basic idea in the $\chi \mathrm{CQM}$ is the fluctuation process where the GBs are emitted by a constituent quark $q^{\uparrow(\downarrow)} \rightarrow \mathrm{GB}^{0}+q^{\prime} \downarrow(\uparrow) \rightarrow\left(q \bar{q}^{\prime}\right)^{0}+q^{\prime} \downarrow(\uparrow)$, where $q \bar{q}^{\prime}+q^{\prime}$ constitute the sea quarks. For the details of the model the reader may refer to Refs. [8].

Apart from the spin of the the constituent quarks and spin of the sea quarks, the magnetic moment of a given baryon in the $\chi \mathrm{CQM}$ also receives contribution from the orbital angular motion of the sea quarks. The total magnetic moment is expressed as [9] $\mu^{p, n}=\mu_{\mathrm{C}}^{p, n}+\mu_{\mathrm{S}}^{p, n}+\mu_{\mathrm{O}}^{p, n}$, where the the contributions of constituent quark spin $\left(\mu_{\mathrm{C}}^{p, n}\right)$, sea quark spin $\left(\mu_{\mathrm{S}}^{p, n}\right)$, and sea orbital $\left(\mu_{\mathrm{O}}^{p, n}\right)$ can be defined in terms of quark magnetic moments and spin polarizations as

$$
\mu_{\mathrm{C}}^{p, n}=\sum_{q=u, d, s} \Delta q_{\mathrm{C}}^{p, n} \mu^{q}, \quad \mu_{\mathrm{S}}^{p, n}=\sum_{q=u, d, s} \Delta q_{\mathrm{S}}^{p, n} \mu^{q}, \quad \mu_{\mathrm{O}}^{p, n}=\sum_{q=u, d, s} \Delta q_{\mathrm{C}}^{p, n} \mu\left(q_{+} \rightarrow\right) .
$$

The $Q^{2}$ dependence of the vector electric and magnetic for factors as well as axial-vector form factors have been experimentally investigated from the PV electron scattering and from the DIS of neutrinos. The conventional dipole form of parametrization has been used to analyse the vector and axial-vector form factors [10]

$$
G_{V, A}^{p, n}\left(Q^{2}\right)=g_{V, A}^{p, n}(0) G_{V, A}^{D}\left(Q^{2}\right)=\frac{g_{V, A}^{p, n}(0)}{\left(1+\frac{Q^{2}}{M_{V, A}^{2}}\right)^{2}}
$$


where the electric and magnetic form factors of the proton and neutron at zero momentum transfer $g_{V}^{p, n}(0)$ for $V=E, M$ correspond to the charge and magnetic moment respectively. $g_{A}^{p}(0)$ and $g_{A}^{n}(0)$ are the isovector axial-vector coupling constants of the proton and neutron corresponding to the axial-vector form factors at zero momentum transfer.

The probabilities of fluctuations to pions, $K, \eta, \eta^{\prime}$ represented by $a, a \alpha^{2}, a \beta^{2}$, and $a \zeta^{2}$ respectively can be calculated in the $\chi \mathrm{CQM}$ at $Q^{2}=0$ after taking into account strong physical considerations and carrying out a fine grained analysis using the well known experimentally measurable spin and flavor distribution functions.

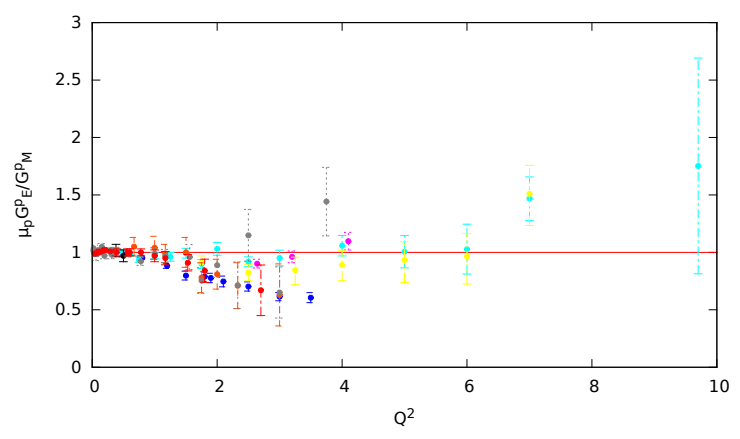

Figure 1: (color online). The ratio $\mu_{p} G_{E}^{p}\left(Q^{2}\right) / G_{M}^{p}\left(Q^{2}\right)$ as a function of $Q^{2}$. The data has been taken from Ref. [11].

In Fig. 1, we have presented the ratio $\mu_{p} G_{E}^{p}\left(Q^{2}\right) / G_{M}^{p}\left(Q^{2}\right)$ corresponding to the electric $\left(G_{E}^{p}\left(Q^{2}\right)\right)$ and magnetic $\left(G_{M}^{p}\left(Q^{2}\right)\right)$ form factors of the proton with $Q^{2}$. A fair agreement with data is obtained. Even though the data varies from $0.95-1.05$, it stays close to 1 . More data for $Q^{2}>4 \mathrm{GeV}^{2}$ may be needed so see if there is some variation from 1 . The proton form factors and their ratios have been measured in the polarization experiments, recoil polarization experiments and beam-target asymmetry measurements $[12,13,14,15]$. The data from different experiments are in general agreement with each other. A real break-through was made towards the understanding of the internal structure of the proton with the measurements of the electromagnetic form factor ratio of the proton, $G_{E}^{p} / G_{M}^{p}$ at $Q^{2}$ is larger than $1 G e V^{2}$ [16]. The data shows a clear deviation of the ratios from unity.

In Fig. 2, we have presented the variation of the ratio $\mu_{n} G_{E}^{n}\left(Q^{2}\right) / G_{M}^{n}\left(Q^{2}\right)$ of the neutron form factors with $Q^{2}$. In Fig. 3, we have presented the ratio $G_{E}^{n}\left(Q^{2}\right) / G_{M}^{n}\left(Q^{2}\right)$. $G_{E}^{n}$ is zero at $Q^{2}=0$ since the neutron has zero charge and the slope at $Q^{2}=0$ is related to the mean-square radius of the neutron. The neutron form factors have been measured in a series of experiments [20], however, in the absence of free neutron targets, the measurements of neutron form factors are more difficult as compared to the measurements of the proton form factors. Our results are in fair agreement with the available experimental data. More data in needed for the profound understanding of the form factors of the neutron.

To summarize, the $\chi \mathrm{CQM}$ helps in the understanding the dynamics of the constituents of the nucleon affected by chiral symmetry breaking in terms of the explicit quark contributions. Further, in light of the precision data available for increased $Q^{2}$ range as well as to present a comprehensive analysis of the vector and axial-vector form factors, the calculations have been extended to anal- 


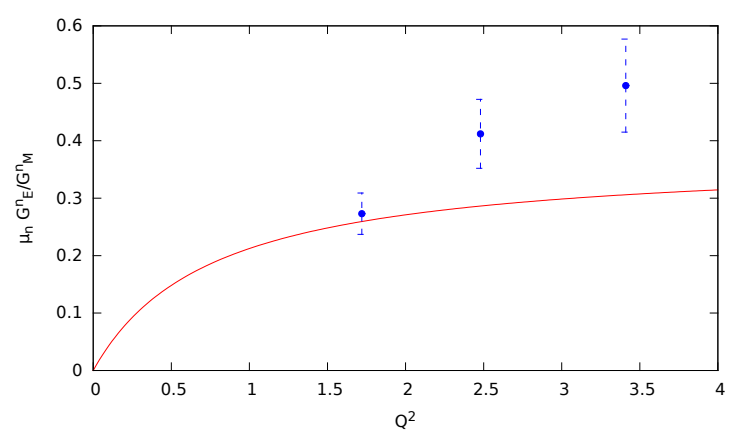

Figure 2: (color online). The ratio $\mu_{n} G_{E}^{n}\left(Q^{2}\right) / G_{M}^{n}\left(Q^{2}\right)$ as a function of $Q^{2}$. The data has been taken from Ref. [17, 18].

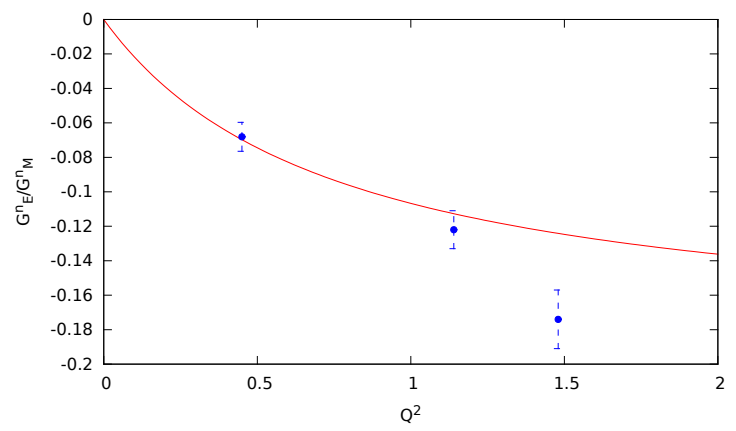

Figure 3: (color online). The ratio $G_{E}^{n}\left(Q^{2}\right) / G_{M}^{n}\left(Q^{2}\right)$ as a function of $Q^{2}$. The data has been taken from Ref. [19].

yse the $Q^{2}$ dependence of these quantities using the conventional dipole form of parametrization. Our results provide important constraints on the future experiments to describe the explicit role of constituent and non-constituent degrees of freedom particularly the strangeness contribution. Different experiments are contemplating the possibility of performing the high precision measurements over a wide $Q^{2}$ region in the near future which will help in the profound understanding of the nonperturbative properties of QCD.

\section{References}

[1] J. Ashman et al. (EMC Collaboration), Phys. Lett. B 206, 364 (1988); J. Ashman et al. (EMC Collaboration), Nucl. Phys. B 328, 1 (1989).

[2] B. Adeva et al. (SMC Collaboration), Phys. Rev. D 58, 112001 (1998); B. Adeva et al. (SMC Collaboration), Phys. Rev. D 60, 072004 (1999).

[3] P. Adams et al., Phys. Rev. D 56, 5330 (1997); P.L. Anthony et al. (E142 Collaboration), Phys. Rev. Lett. 71, 959 (1993); K. Abe et al. (E143 Collaboration), Phys. Rev. Lett. 76, 587 (1996); K. Abe et al. (E154 Collaboration), Phys. Rev. Lett. 79, 26 (1997).

[4] A. Airapetian et al. (HERMES Collaboration), Phys. Rev. D 71, 012003 (2005); A. Airapetian et al. (HERMES Collaboration), Phys. Rev. D 75, 012007 (2007). 
[5] L.A. Ahrens et al., Phys. Rev. D 35, 785 (1987).

[6] S. Brice et al., A Proposal for a near detector experiment on the booster neutrino beamline: FINeSSE, hep-ex/0402007.

[7] S.F. Pate, Phys. Rev. Lett. 92, 082002 (2004).

[8] H. Dahiya and M. Gupta, Phys. Rev. D 78, 014001 (2008); H. Dahiya and M. Randhawa, Phys. Rev. D 90, 074001 (2014); H. Dahiya, Phys. Rev. D 91, 094010 (2015); A. Girdhar, H. Dahiya and M. Randhawa, Phys. Rev. D 92, 033012 (2015); H. Dahiya and M. Randhawa, Phys. Rev. D 93, 114030 (2016).

[9] S. Weinberg, Physica A 96, 327 (1979); A. Manohar and H. Georgi, Nucl. Phys. B 234, 189 (1984); E.J. Eichten, I. Hinchliffe, and C. Quigg, Phys. Rev. D 45, 2269 (1992).

[10] D.H. Beck and R.D. McKeown, Annual Rev. of Nucl. and Part. Sc. 51, 189 (2001).

[11] B.D. Milbrath et al. (Bates FPP Collaboration), Phys. Rev. Lett. 80, 452 (1998); Erratum-ibid: Phys. Rev. Lett. 82, 2221 (1999); V. Punjabi, C.F. Perdrisat et al., Phys. Rev. C 71, 055202 (2005); Erratum-ibid: Phys. Rev. C 71, 069902 (2005); V. Punjabi, C.F. Perdrisat et al., Eur. Phys. J. A 51, 79 (2015).

[12] M.K. Jones et al. (Jefferson Lab Hall A Collaboration), Phys. Rev. Lett. 84, 1398 (2000); O. Gayou et al. (Jefferson Lab Hall A Collaboration), Phys. Rev. Lett. 88, 092301 (2002).

[13] G. Warren et al. (Jefferson Lab E93-026 Collaboration), Phys. Rev. Lett. 92, 042301 (2004); S. Riordan, S. Abrahamyan, B. Craver, A. Kelleher, A. Kolarkar et al., Phys. Rev. Lett. 105, 262302 (2010).

[14] B. Hu et al., Phys. Rev. C 73, 064004 (2006); M. Paolone, S. Malace, S. Strauch, I. Albayrak, J. Arrington et al., Phys. Rev. Lett. 105, 072001 (2010); G. Ron et al. (Jefferson Lab Hall A Collaboration), Phys. Rev. C 84, 055204 (2011); X. Zhan, K. Allada, D. Armstrong, J. Arrington, W. Bertozzi et al., Phys. Lett. B 705, 59 (2011).

[15] M. Jones et al. (Resonance Spin Structure Collaboration), Phys. Rev. C 74, 035201 (2006); C.B. Crawford et al., Phys. Rev. Lett. 98, 052301 (2007).

[16] A. Puckett, E. Brash, M. Jones, W. Luo, M. Meziane et al., Phys. Rev. Lett. 104, 242301 (2010); A. Puckett, E. Brash, O. Gayou, M. Jones, L. Pentchev et al., Phys. Rev. C 85, 045203 (2012).

[17] R. Schiavilla and I. Sick, Phys. Rev. C 64, 041002 (2001); S. Riordan, S. Abrahamyan et al., Phys. Rev. Lett. 105, 262302 (2010).

[18] J. Becker et al., Eur. Phys. J. A 6, 329 (1999); B.S. Schlimme et al., Phys. Rev. Lett. 111, 132504 (2013).

[19] B. Plaster, A.Yu. Semenov et al. (Jefferson Laboratory E93-038 Collaboration), Phys. Rev. C 73, 025205 (2006).

[20] H. Anklin et al., Phys. Lett. B 428, 248 (1998); G. Kubon et al., Phys. Lett. B 524, 26 (2002); B. Anderson et al. (Jefferson Lab E95-001 Collaboration), Phys. Rev. C 75, 034003 (2007).

[21] A.A. Aguilar-Arevalo et al. (MiniBooNE Collaboration), Phys. Rev. D 82, 092005 (2010); T. Golan, K.M. Graczyk, C. Juszczak, J.T. Sobczyk, Phys. Rev. C 88, 024612 (2013).

[22] R.C. Walker et al., Phys. Rev. D 49, 5671 (1994); L. Andivahis et al., Phys. Rev. D 50, 5491 (1994); I.A. Qattan et al., Phys. Rev. Lett. 94, 142301 (2005); Y. Liang, M.E. Christy, R. Ent, C.E. Keppel, Phys. Rev. C 73, 065201 (2006).

[23] A.F. Sill et al., Phys. Rev. D 48, 29 (1993). 